\title{
A Novel Exon 3 Mutation (P66S) in the SOD1 Gene in Familial ALS
}

\author{
Wonki Baek, Seong-Ho Koh, Young Seo Kim, Hyun Young Kim, Min-Jung Kwon, Chang-Seok Ki, \\ Seung Hyun Kim
}

Can J Neurol Sci. 2012; 39: 245-246

Approximately $10 \%$ of cases of amyotrophic lateral sclerosis (ALS) are familial, with up to $20 \%$ caused by mutations in the superoxide dismutase-1 gene (SOD1), a ubiquitously expressed free-radical scavenging enzyme. ${ }^{1}$ At the time of writing, about 150 SOD1 gene mutations have been reported. The SOD1 gene is composed of five exons and four introns. Different mutations account for the variable severity of ALS. Moreover, the clinical course is quite variable due to mutations in different exons. For example, Codon4 (A4V) of exon 1, responsible for $50 \%$ of the SOD1 mutations associated with ALS in North America, is an aggressive form of ALS with a survival time of less than two years after disease onset. Although, only about ten mutations in exon 3 have been reported, some of the mutations are associated with slow progression. ${ }^{2}$

Here, we describe a family with a novel exon 3 mutation (P66S) in the SOD1 gene with slow progression.

\section{Case Reports}

A 44-year-old woman presented with a five year history of progressive leg weakness followed by arm weakness. At 39 years-of-age, she first noticed weakness in her lower limbs such that she had difficulty climbing stairs. She became wheelchairdependent within the next year and by the end of two years the weakness had progressed to her hands. She was examined at age 44.

Her past medical and social histories were uninformative. She had four siblings and they were all asymptomatic. She denied previous neck or back trauma, and cervical spinal magnetic resonance imaging (MRI) was normal. Her cognitive function was normal on neuropsychological testing and there were no abnormalities on cranial nerve examination. In the rest of the physical examination there was atrophy of the intrinsic hand muscles and legs bilaterally. She had mild weakness of both distal hands (Medical Research Council $(\mathrm{MRC})=4 / 5)$ and of both arms $(\mathrm{MRC}=4 / 5)$, and weakness in both lower limbs $(\mathrm{MRC}=3 / 5)$ with atrophic changes in both thenar muscles. Generalized fasciculations were present in upper and lower limbs. Deep tendon reflexes were hyperreflexic. There was clonus, and Babinski and Hoffmann signs were present. Sensory and cerebellar functions were normal. The patient had the clinical features of relatively slow progression. She could walk with assistance despite the fact that the weakness had begun four years earlier.

Electrophysiologic studies revealed active denervation and regenerative potentials in three regions (cervical, thoracic and lumbosacral) with decreased CMAP amplitudes in motor nerve conduction studies and normal sensory conduction velocities, compatible with ALS. Increased motor evoked potential threshold and central motor conduction time were observed in transcranial motor evoked potential (MEP). To test for the presence of a SOD1 mutation, DNA sequencing was performed.

\subsection{Mutation analysis of the SOD1 gene}

After obtaining informed consent, we carried out genetic studies of the ALS patient. After the presence of the SOD1 mutation was confirmed, we created a three generation pedigree including the other seven members of the family, after obtaining consent for genetic studies. Genomic DNA was isolated from peripheral blood leukocytes using a Wizard genomic DNA purification kit (Promega, Madison, WI). All five SOD1coding exons and their flanking intronic regions were amplified by polymerase chain reaction (PCR) using primers designed by the authors (available upon request). Cycle sequencing was carried out using a BigDye Terminator Cycle Sequencing Ready Reaction kit (Applied Biosystems, Foster City, CA) on an ABI 3100 Genetic Analyzer (Applied Biosystems).

\subsection{Results of genetic analysis}

Direct sequencing of the SOD1 gene in the proband revealed a heterozygous cytosine to thymine ( $\mathrm{C}$ to $\mathrm{T}$ ) transition at cDNA position 205, which converted proline to serine at amino acid position $66(\mathrm{P} 66 \mathrm{~S})$. This mutation has not been reported previously and was not observed in 600 control chromosomes. Therefore, further DNA analysis of several family members was performed and revealed that the proband's mother (I-2), younger brother (II-3) and son (III-1) had the same mutation (Figure). To find out whether the P66S substitution is likely to be harmful, in silico prediction of the functional consequence was performed using SIFT (Sorting Intolerant from Tolerant; http:// blocks.fhcrc.org/sift/SIFT.html), PANTHER (http://www. pantherdb.org), and PolyPhen (Polymorphism Phenotyping,

\footnotetext{
From the Department of Neurology (WB, S-HK, YSK, HYK, SHK), Hanyang University College of Medicine; Department of Laboratory Medicine (M-JK), Kangbuk Samsung Hospital, Sungkyunkwan University School of Medicine; Department of Laboratory Medicine and Genetics (C-SK), Samsung Medical Center, Sungkyunkwan University School of Medicine, Hanyang University Hospital, Seoul, South Korea.

Received August 29, 2011. Final Revisions Submitted October 28, 2011. Correspondence to: Seung Hyun Kim, Department of Neurology, Hanyang University Hospital, 17 Haengdang-dong, Seongdong-gu, Seoul 133-792, South Korea.
} 


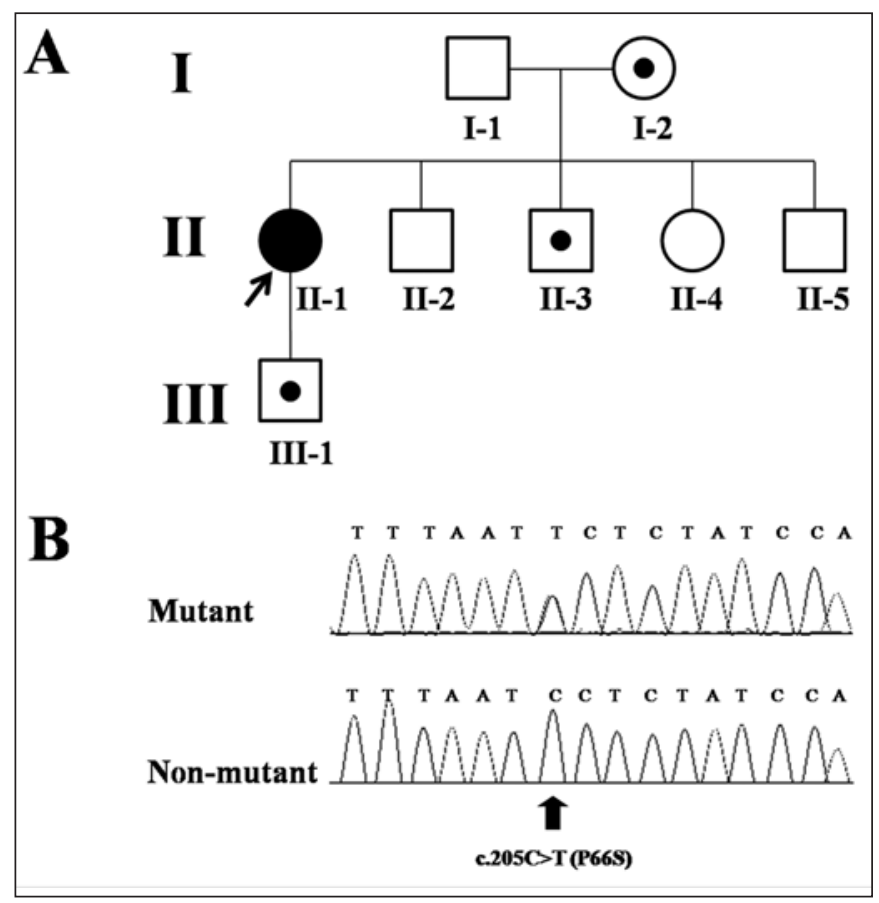

Figure: A. Pedigree of the family with ALS showing one affected subject (proband, 44-years-old) and three asymptomatic obligate carriers (I-2, II-3, III-1).; (I-2: 66-years-old, II-3: 42-years-old, III-1: 22 years old) B. Result of SOD1 gene mutation analysis showing mutation in exon 3 of the SOD1 gene, a C-to-T transversion at nucleotide position 205 leading to a P66S amino acid change.

http://coot.embl.de/PolyPhen). P66S was predicted by these websites to be not-tolerated, deleterious, and probably damaging, respectively. Moreover, orthologous conservation information from Evolutionary annotation database version 7.5 (Evola, http://www.h-invitational.jp/evola_main/annotation.cgi) revealed that the P66 residue is highly conserved across species.

The proband was the only affected member while the three others were asymptomatic on neurologic exam and electrophysiological tests. Her mother had not yet developed symptoms even though she was 65 .

\section{DisCUSSION}

Mutations of the SOD1 gene in ALS were first identified by Rosen et al. ${ }^{1}$ Several observations suggest that pathogenic effects of $\mathrm{Cu} / \mathrm{Zn}$-SOD mutations in familial ALS do not result from loss of enzymatic dismutase function, but instead from gain of an adverse property by the mutant protein. ${ }^{3}$ Apparently, the mutation converts SOD1 from an anti-apoptotic gene to a proapoptotic one.

The SOD1 gene has five exons and four introns that encode a protein with 153 amino acids. Exons 4 and 5 are considered to be frequent sites of mutation, while mutations in exon 3 are relatively rare. Over $60 \%$ of all SOD1 mutations have been mapped to exon 4 or 5 ; only about ten exon 3 cases have been reported at the time of writing. ${ }^{4}$ Several explanations have been suggested for the low frequency of mutations in this exon. (1) Exon 3 may be critical for function, and mutations would be lethal in utero, (2) Mutations in exon 3 are associated with reduced penetrance or a benign clinical course, (3) For technical reasons (inadequate PCR primer sets and/or single stranded confirmation polymorphism running conditions) there are obstacles to detecting exon 3 mutations. ${ }^{2}$

The effect of the P66S mutation was similar to that of the slowly progressing D76V mutation. ${ }^{2}$ However, this mutation has one unique clinical feature: while almost all the mutations in exon 3 are associated with predominantly lower motor neuron signs ${ }^{4}$ the patient with the P66S mutation displayed upper motor neuron signs on both upper and lower extremities. There is some evidence that disease phenotype and progression can be influenced by epigenetic factors such as gender, modifier genes, environmental influences, and other unknown agents. ${ }^{5}$ However, the mutation site also plays an important role in determining the phenotype. Indeed, a patient with SOD1 mutation (P66S in exon3)-mediated ALS presented with relatively slow progression. Investigations of penetrance of this mutation are essential to future research.

\section{ACKNoWledgments / Conflicts OF INTEREST}

This study was supported by a grant from the Korean Health Technology R\&D Project, Ministry for Health, Welfare \& Family Affairs, Republic of Korea. (A101712)

The funding organization had no role in the genetic analysis, or in the preparation of this report.

Dr. Seung-Hyun Kim received research grants from the Korean Health Technology R\&D Project, Ministry for Health, Welfare \& Family Affairs, Republic of Korea.

\section{REFERENCES}

1. Rosen DR. Mutations in $\mathrm{Cu} / \mathrm{Zn}$ superoxide dismutase gene are associated with familial amyotrophic lateral sclerosis. Nature. 1993;364:362.

2. Segovia-Silvestre T, Andreu AL, Vives-Bauza C, Garcia-Arumi E, Cervera C, Gamez J. A novel exon 3 mutation (D76V) in the SOD1 gene associated with slowly progressive ALS. Amyotroph Lateral Scler Other Motor Neuron Disord. 2002;3:69-74.

3. Giess R, Holtmann B, Braga M, et al. Early onset of severe familial amyotrophic lateral sclerosis with a SOD-1 mutation: potential impact of CNTF as a candidate modifier gene. Am J Hum Genet. 2002;70:1277-86.

4. del Grande A, Luigetti M, Conte A, et al. A novel L67P SOD1 mutation in an Italian ALS patient. Amyotroph Lateral Scler. 2011;12:150-2.

5. Radunovic A, Leigh $\mathrm{PN}$. $\mathrm{Cu} / \mathrm{Zn}$ superoxide dismutase gene mutations in amyotrophic lateral sclerosis: correlation between genotype and clinical features. J Neurol Neurosurg Psychiatry. 1996;61:565-72. 